\title{
Bypass schlägt Stent
}

\author{
Die Frage, welche Methode der Re- \\ vaskularisierung für Diabetiker mit \\ fortgeschrittener Koronarerkrankung \\ die Beste ist, ist in der FREEDOM- \\ Studie definitiv geklärt worden: Die \\ koronare Bypass-Operation ist der \\ Stent-Behandlung klar überlegen - \\ auch in puncto Mortalitätssenkung.
}

- Die Diskussion darüber, ob bei koronarer Mehrgefäßerkrankung die BypassOp. oder der Koronarstent die beste Option ist, erhitzt die Gemüter von Herzchirurgen und interventionellen Kardiologen schon lange. Dabei ging es nicht zuletzt um die Gruppe der Diabetiker, bei denen infolge einer diffusen Koronarsklerose oft eine Mehrgefäßerkrankung mit komplexen Läsionen besteht.

Für die FREEDOM-Studie wurden 1900 Diabetiker mit angiografisch gesi- cherter koronarer Mehrgefäßerkrankung - darunter $83 \%$ mit Dreigefäßerkrankung - ausgewählt. Alle waren auf eine aggressive medikamentöse Therapie eingestellt und wurden dann entweder einer Bypass-Op. oder einer perkutanen Koronarintervention (PCI) mit Implantation von Drug-eluting Stents (DES) unterzogen.

\section{Signifikanter Unterschied auch bei Mortalität}

Die Bilanz nach fünf Jahren: Mit 18,7 vs. 26,6\% war die Gesamtrate der primären Endpunktereignisse (Tod, Myokardinfarkt, Schlaganfall) in der Bypass-Gruppe signifikant niedriger als in der PCIGruppe. Den Ausschlag dafür gab eine jeweils signifikante relative Reduktion sowohl der Herzinfarktrate (6,0 vs. $13,9 \%)$ als auch der Gesamtsterberate
(10,9 vs. 16,3\%) durch die Bypass-Op. Ein Unterschied zugunsten der chirurgischen Behandlung wurde erstmals nach etwa zwei Jahren erkennbar, danach ging die Schere zwischen beiden Gruppen bei der Ereignisrate immer weiter auseinander. Die Bypass-Op. war allerdings im Vergleich zur PCI mit einem erhöhten Risiko für Schlaganfälle assoziiert, wobei die meisten Ereignisse innerhalb der ersten 30 Tage nach der Operation auftraten. In der Nettobilanz überwog dennoch klar der Nutzen dieser Therapie. Bei der kardiovaskulären Mortalität ergab sich nur ein nicht signifikanter Trend zugunsten der koronarchirurgischen Behandlung (6,8 vs. $10,9 \%)$.

Peter OVerbeck -

- Quelle: AHA-Kongress, 4.11.2012,

Farkouh ME, et al. N Engl J Med 2012, doi:

10.1056/NEJMoa1211585

\section{Erstaunlich wirksam bei stabiler KHK}

\section{Colchicin verhindert Herzattacken}

\section{Colchicin entwickelt sich zum Herz- medikament: Nachdem es kürzlich in einer Studie nach Kardiochirurgie Vorhofflimmern verhütete, punktet es nun bei stabiler KHK: In der LoDo- Co-Studie reduzierte es das Risiko für akute Herzattacken um über $70 \%$.}

- LoDoCo steht für „Low Dose Colchicine for Secondary Prevention of Cardiovascular Disease“. Die Ergebnisssse dieser prospektiven, randomisierten Studie wurden von Dr. M. Nixdorf vom Heart Research Institute in Perth vorgestellt.

Warum Colchicin bei KHK? Nixdorf erklärte, dass sich in den Koronarplaques von ACS-Patienten aktivierte neutrophile Granulozyten befinden. Werden diese gehemmt, könnte dies die Plaque-Stabilität erhöhen und das Risi- ko für koronare Ereignisse reduzieren. Colchicin wirkt breit antiinflammatorisch und inhibiert besonders die durch neutrophile Granulozyten vermittelte Entzündung - additiv zu einer Therapie mit ASS und Statinen. Trotz gelegentlicher gastrointestinaler Nebenwirkungen ist es in der Langzeitanwendung sicher.

In die LoDoCo-Studie wurden 532 Patienten mit stabiler KHK einbezogen, von denen 282 mit täglich $0,5 \mathrm{mg}$ Colchicin behandelt wurden. Nahezu alle nahmen Thrombozytenfunktionshemmer und Statine ein. Primärer Endpunkt waren akute Koronarsyndrome, Herzstillstand oder nicht kardioembolische ischämische Schlaganfälle.

Nach einer medianen Behandlungszeit von drei Jahren hatten 40/250 (16\%) der Kontrollpatienten, aber nur 15/282
(5,3\%) Patienten in der Colchicin-Gruppe einen primären Endpunkt erlitten $(\mathrm{p}<0,001)$. Reduziert wurde insbesondere das Risiko für neue akute Koronarsyndrome an anderen Stellen als den bisherigen Stent-Lokalisationen (30 vs. 9 Ereignisse, HR: 0,21). Sowohl das Risiko für STEMI als auch für instabile Angina sank. Fünf Patienten der Kontrollgruppe und kein Patient der Colchicin-Gruppe erlitt einen Herztod. Vier Patienten der Kontrollgruppe und ein Patient der Colchicin-Gruppe bekam einen ischämischen Schlaganfall.

Die Wirksamkeit, so Studienautor Nixdorf, stellte sich sehr früh nach Behandlungsbeginn ein. 16\% setzten Colchicin aufgrund gastrointestinaler Beschwerden ab. DR. MED. DIRK EINECKE - Quelle: AHA-Kongress 2012 